\title{
Fractional complex transform method for wave equations on Cantor sets within local fractional differential operator
}

\author{
Wei-Hua Su ${ }^{1,2}$, Xiao-Jun Yang ${ }^{3,4^{*}}$, H Jafari $^{5}$ and Dumitru Baleanu $u^{6,7,8}$
}

"Correspondence:

dyangxiaojun@163.com

${ }^{3}$ School of Mechanics \& Civil

Engineering, China University of

Mining \& Technology, Xuzhou,

Jiangsu 221008, China

${ }^{4}$ Institute of Software Science,

Zhengzhou Normal University,

Zhengzhou, 450044, China

Full list of author information is

available at the end of the article

\begin{abstract}
This paper points out the fractional complex transform method for wave equations on Cantor sets within the local differential fractional operators. The proposed method is efficient to handle differential equations on Cantor sets.
\end{abstract}

Keywords: fractional complex transform method; wave equations; local fractional differential operators; Cantor sets

\section{Introduction}

In recent years, the local fractional calculus has attracted a lot of interest for scientists and engineers. Several sections of local fractional derivative had been introduced, i.e. the local fractional derivative structured by Kolwankar and Gangal [1-5], the modified RiemannLiouville derivative given by Jumarie [3-7], the fractal derivative suggested by Parvate and Gangal [3-5, 8, 9], the fractal derivative considered by Chen et al. [3-5, 10-12], the generalized fractal derivative proposed Chen et al. [12], the local fractional derivative presented by Adda and Cresson [3-5, 13], the fractal derivative suggested by He [3-5, 14-16] and the local fractional derivative structured in [3-5, 17-25]. As a result, the local fractional calculus theory become important for modelling problems for fractal mathematics and engineering on Cantor sets and it plays a key role in many applications in several fields such as the theorical physics $[2,3]$, the heat conduction theory $[3,14,17]$, the fracture and elasticity mechanics $[3,19]$, the fluid mechanics $[3,26]$, signal analysis $[4,5]$, Fourier and wavelet analysis $[4,5]$, tensor analysis [3], complex analysis [4, 5], optimization method [3] and so on. For example, the local fractional Fokker-Planck equation was proposed in [2]. The fractal heat conduction problems were presented $[3,14]$. The principles of virtual work, minimum potential and complementary energy in the mechanics of fractal media were investigated in $[3,19,25]$. The fluid flow in fractal porous medium was studied in [26]. The diffusion problems in fractal media was reported in [11, 24, 27]. Authors of [28] were concerned with one-dimension wave equation on Cantor sets, which read

$$
\frac{\partial^{2 \alpha} u(x, t)}{\partial t^{2 \alpha}}+a^{2 \alpha} \frac{\partial^{2 \alpha} u(x, t)}{\partial x^{2 \alpha}}=0,
$$

o 2013 Su et al.: licensee Springer. This is an Open Access article distributed under the terms of the Creative Commons Attribution License (http://creativecommons.org/licenses/by/2.0), which permits unrestricted use, distribution, and reproduction in any medium, provided the original work is properly cited. 
where the operator was described as local fractional operator given by [3-5, 17-23]

$$
f^{(\alpha)}\left(x_{0}\right)=\left.\frac{d^{\alpha} f(x)}{d x^{\alpha}}\right|_{x=x_{0}}=\lim _{x \rightarrow x_{0}} \frac{\Delta^{\alpha}\left(f(x)-f\left(x_{0}\right)\right)}{\left(x-x_{0}\right)^{\alpha}}
$$

with $\Delta^{\alpha}\left(f(x)-f\left(x_{0}\right)\right) \cong \Gamma(1+\alpha) \Delta\left(f(x)-f\left(x_{0}\right)\right)$ and where local fractional operator of $k$ th order was [3-5]

$$
\frac{\partial^{k \alpha} f(x)}{\partial x^{k \alpha}}=\overbrace{\frac{\partial^{\alpha}}{\partial x^{\alpha}} \cdots \frac{\partial^{\alpha}}{\partial x^{\alpha}}}^{k \text { times }} f(x) .
$$

However, it has not been extended to two-dimensional (2-D) and three-dimensional (3-D) wave equations on Cantor sets due to the structural behavior of complexity of Cantor materials.

Fractional complex transform method via the modified Riemann-Liouville derivative [3-7] was first proposed in 2010 [29-31] and was applied to model the heat conduction problem and differential equations [15, 32-34]. The fractional complex transform met some problems in applications when the modified Riemann-Liouville derivative [3-7] was adopted due to the complex chain rule [34]. Fractional complex transform [17, 35] via the local fractional derivative was first proposed by the following chain rule [3-5]

$$
\frac{d^{\alpha} y(x)}{d x^{\alpha}}=f^{(1)}(g(x)) g^{(\alpha)}(x)
$$

where there exist $f^{(1)}(g(x))$ and $g^{(\alpha)}(x)$. The heat conduction problem in fractal media was processed by the fractional complex transform within local fractional derivative [17]. The similar results, but using different operators, can be seen in [36-38].

In this work, we consider 3-D wave equation on Cantor sets described by the local fractional derivative suggested in $[3-5,17-25]$, which is written in the form

$$
\frac{\partial^{2 \alpha} u(x, y, z, t)}{\partial t^{2 \alpha}}+a^{2 \alpha} \nabla^{2 \alpha} u(x, y, z, t)=0
$$

where local fractional Laplace operator is noted by $[3-5,39]$

$$
\nabla^{2 \alpha}=\frac{\partial^{2 \alpha}}{\partial x^{2 \alpha}}+\frac{\partial^{2 \alpha}}{\partial y^{2 \alpha}}+\frac{\partial^{2 \alpha}}{\partial z^{2 \alpha}}
$$

The 2-D wave equation on Cantor sets can easily been obtained

$$
\frac{\partial^{2 \alpha} u(x, y, t)}{\partial t^{2 \alpha}}+a^{2 \alpha}\left(\frac{\partial^{2 \alpha} u(x, y, t)}{\partial x^{2 \alpha}}+\frac{\partial^{2 \alpha} u(x, y, t)}{\partial y^{2 \alpha}}\right)=0 .
$$

Our attention is focused on convert the 3-D wave equation on Cantor sets by using the fractional complex transform method via local fractional derivatives.

The organization of this manuscript is given below: In Section 2, the fractional complex transform method via local fractional derivatives is presented. Section 3 shows the applications of fractional complex transform to convert 3-D and 2-D wave equations on Cantor sets. Conclusions end the manuscript in Section 4. 


\section{Fractional complex transform method via local fractional derivatives}

In this section, we consider the fractional complex transform method for differential equations on Cantor sets [17, 35].

\section{Proposition 1 If}

$$
\left\{\begin{array}{l}
X=\frac{x^{\alpha}}{\Gamma(1+\alpha)} \\
Y=\frac{y^{\alpha}}{\Gamma(1+\alpha)}
\end{array}\right.
$$

where $0<\alpha \leq 1$, then we have

$$
\frac{\partial^{\alpha} U_{1}(x, y)}{\partial x^{\alpha}}+\frac{\partial^{\alpha} U_{2}(x, y)}{\partial y^{\alpha}}=0
$$

such that

$$
\frac{\partial U_{1}(X, Y)}{\partial X}+\frac{\partial U_{2}(X, Y)}{\partial Y}=0
$$

where there exist the relations

$$
\frac{\partial U_{1}(X, Y)}{\partial X}, \frac{\partial U_{2}(X, Y)}{\partial Y}, \frac{\partial^{\alpha} U_{1}(x, y)}{\partial x^{\alpha}}, \frac{\partial^{\alpha} U_{2}(x, y)}{\partial y^{\alpha}} .
$$

Proof Let us consider the fractional complex transform (8), then we can write

$$
\frac{\partial^{\alpha} U_{1}(x, y)}{\partial x^{\alpha}}=\frac{\partial U_{1}(X, Y)}{\partial X} \frac{\partial^{\alpha} X}{\partial x^{\alpha}}+\frac{\partial U_{1}(X, Y)}{\partial Y} \frac{\partial^{\alpha} Y}{\partial x^{\alpha}}=\frac{\partial U_{1}(X, Y)}{\partial X}
$$

and finally we conclude

$$
\frac{\partial^{\alpha} U_{2}(x, y)}{\partial y^{\alpha}}=\frac{\partial U_{2}(X, Y)}{\partial Y} \frac{\partial^{\alpha} Y}{\partial y^{\alpha}}+\frac{\partial U_{2}(X, Y)}{\partial X} \frac{\partial^{\alpha} Y}{\partial X^{\alpha}}=\frac{\partial U_{2}(X, Y)}{\partial Y}
$$

respectively.

Proposition 2 Assuming that (8) is valid, then we can transfer

$$
\frac{\partial^{2 \alpha} U_{2}(x, y)}{\partial x^{2 \alpha}}+\frac{\partial^{2 \alpha} U_{1}(x, y)}{\partial x^{\alpha} \partial y^{\alpha}}+\frac{\partial^{2 \alpha} U_{1}(x, y)}{\partial y^{\alpha} \partial x^{\alpha}}+\frac{\partial^{2 \alpha} U_{2}(x, y)}{\partial y^{2 \alpha}}=0
$$

into

$$
\frac{\partial^{2} U_{2}(X, Y)}{\partial X^{2}}+\frac{\partial^{2} U_{1}(X, Y)}{\partial X \partial Y}+\frac{\partial^{2} U_{2}(X, Y)}{\partial Y \partial X}+\frac{\partial^{2} U_{2}(X, Y)}{\partial Y^{2}}=0
$$

where there exist the following relations:

$$
\begin{aligned}
& \frac{\partial U_{1}(X, Y)}{\partial X}, \frac{\partial U_{2}(X, Y)}{\partial Y}, \frac{\partial^{2} U_{1}(X, Y)}{\partial X^{2}}, \frac{\partial^{2} U_{1}(X, Y)}{\partial Y \partial X}, \frac{\partial^{2} U_{2}(X, Y)}{\partial X \partial Y}, \frac{\partial^{2} U_{2}(X, Y)}{\partial Y^{2}}, \\
& \frac{\partial^{\alpha} U_{1}(x, y)}{\partial x^{\alpha}}, \frac{\partial^{\alpha} U_{2}(x, y)}{\partial y^{\alpha}}, \frac{\partial^{2 \alpha} U_{1}(x, y)}{\partial x^{2 \alpha}}, \frac{\partial^{2 \alpha} U_{1}(x, y)}{\partial y^{\alpha} \partial x^{\alpha}}, \frac{\partial^{2 \alpha} U_{2}(x, y)}{\partial x^{\alpha} \partial y^{\alpha}}, \frac{\partial^{2 \alpha} U_{2}(x, y)}{\partial y^{2 \alpha}} .
\end{aligned}
$$


Proof Taking the Cantorian coordination pairs (8) yields

$$
\frac{\partial^{2 \alpha} U_{1}(x, y)}{\partial x^{2 \alpha}}=\frac{\partial^{2} U_{1}(X, Y)}{\partial X^{2}} \frac{\partial^{\alpha} X}{\partial x^{\alpha}}+\frac{\partial^{2} U_{1}(X, Y)}{\partial Y^{2}} \frac{\partial^{\alpha} Y}{\partial x^{\alpha}}=\frac{\partial^{2} U_{1}(X, Y)}{\partial X^{2}} .
$$

In a similar manner as in the previous proposition, we obtain

$$
\begin{aligned}
& \frac{\partial^{2 \alpha} U_{2}(x, y)}{\partial y^{2 \alpha}}=\frac{\partial^{2} U_{2}(X, Y)}{\partial Y^{2}} \frac{\partial^{\alpha} Y}{\partial y^{\alpha}}+\frac{\partial^{2} U_{2}(X, Y)}{\partial Y^{2}} \frac{\partial^{\alpha} X}{\partial y^{\alpha}}=\frac{\partial^{2} U_{2}(X, Y)}{\partial X^{2}}, \\
& \frac{\partial^{2 \alpha} U_{1}(x, y)}{\partial y^{\alpha} \partial x^{\alpha}}=\frac{\partial^{2} U_{1}(X, Y)}{\partial X \partial Y} \frac{\partial^{\alpha} Y}{\partial y^{\alpha}}+\frac{\partial^{2} U_{1}(X, Y)}{\partial X \partial Y} \frac{\partial^{\alpha} Y}{\partial x^{\alpha}}=\frac{\partial^{2} U_{1}(X, Y)}{\partial Y \partial X}, \\
& \frac{\partial^{2 \alpha} U_{2}(x, y)}{\partial^{\alpha} x \partial y^{\alpha}}=\frac{\partial^{2} U_{2}(X, Y)}{\partial Y \partial X} \frac{\partial^{\alpha} X}{\partial x^{\alpha}}+\frac{\partial^{2} U_{2}(X, Y)}{\partial X \partial X} \frac{\partial^{\alpha} X}{\partial y^{\alpha}}=\frac{\partial^{2} U_{2}(X, Y)}{\partial X \partial Y} .
\end{aligned}
$$

Therefore, we conclude that

$$
\frac{\partial^{2} U_{2}(X, Y)}{\partial X^{2}}+\frac{\partial^{2} U_{1}(X, Y)}{\partial X \partial Y}+\frac{\partial^{2} U_{2}(X, Y)}{\partial Y \partial X}+\frac{\partial^{2} U_{2}(X, Y)}{\partial Y^{2}}=0
$$

Proposition 3 Let us assume that there is the fractional complex transform

$$
\left\{\begin{array}{l}
X=\frac{x^{\alpha}}{\Gamma(1+\alpha)}, \\
Y=\frac{y^{\alpha}}{\Gamma(1+\alpha)}, \\
Z=\frac{z^{\alpha}}{\Gamma(1+\alpha)}
\end{array}\right.
$$

and

$$
\frac{\partial U_{1}(x, y, z)}{\partial x}+\frac{\partial U_{2}(x, y, z)}{\partial y}+\frac{\partial U_{3}(x, y, z)}{\partial z}=0,
$$

then

$$
\frac{\partial^{\alpha} U_{1}(x, y, z)}{\partial x^{\alpha}}+\frac{\partial^{\alpha} U_{2}(x, y, z)}{\partial y^{\alpha}}+\frac{\partial^{\alpha} U_{3}(x, y, z)}{\partial z^{\alpha}}=0 .
$$

Proof By using the fractional complex transform (19), we obtain

$$
\begin{aligned}
& \frac{\partial^{\alpha} U_{1}(x, y, z)}{\partial x^{\alpha}} \\
& =\frac{\partial U_{1}(X, Y, Z)}{\partial X} \frac{\partial^{\alpha} X}{\partial x^{\alpha}}+\frac{\partial U_{1}(X, Y, Z)}{\partial Y} \frac{\partial^{\alpha} Y}{\partial x^{\alpha}}+\frac{\partial U_{1}(X, Y, Z)}{\partial Z} \frac{\partial^{\alpha} Z}{\partial x^{\alpha}} \\
& =\frac{\partial U_{1}(X, Y, Z)}{\partial X}, \\
& \frac{\partial^{\alpha} U_{2}(x, y, z)}{\partial y^{\alpha}} \\
& =\frac{\partial U_{2}(X, Y, Z)}{\partial X} \frac{\partial^{\alpha} X}{\partial y^{\alpha}}+\frac{\partial U_{2}(X, Y, Z)}{\partial Y} \frac{\partial^{\alpha} Y}{\partial y^{\alpha}}+\frac{\partial U_{2}(X, Y, Z)}{\partial Z} \frac{\partial^{\alpha} Z}{\partial y^{\alpha}} \\
& =\frac{\partial U_{2}(X, Y, Z)}{\partial Y},
\end{aligned}
$$




$$
\begin{aligned}
& \frac{\partial^{\alpha} U_{3}(x, y, z)}{\partial z^{\alpha}} \\
& \quad=\frac{\partial U_{3}(X, Y, Z)}{\partial X} \frac{\partial^{\alpha} X}{\partial z^{\alpha}}+\frac{\partial U_{3}(X, Y, Z)}{\partial Y} \frac{\partial^{\alpha} Y}{\partial z^{\alpha}}+\frac{\partial U_{3}(X, Y, Z)}{\partial Z} \frac{\partial^{\alpha} Z}{\partial z^{\alpha}} \\
& =\frac{\partial U_{3}(X, Y, Z)}{\partial X} .
\end{aligned}
$$

Clearly, from Eqs. (23)-(24), we directly obtain (21).

Proposition 4 Let us consider the following fractional complex transform (19) and

$$
\frac{\partial^{2} U_{1}(x, y, z)}{\partial x^{2}}+\frac{\partial^{2} U_{2}(x, y, z)}{\partial y^{2}}+\frac{\partial^{2} U_{3}(x, y, z)}{\partial z^{2}}=0,
$$

thus, we obtain

$$
\frac{\partial^{2 \alpha} U_{1}(x, y, z)}{\partial x^{2 \alpha}}+\frac{\partial^{2 \alpha} U_{2}(x, y, z)}{\partial y^{2 \alpha}}+\frac{\partial^{2 \alpha} U_{3}(x, y, z)}{\partial z^{2 \alpha}}=0 .
$$

Proof Applying the fractional complex transform method (19), we obtain the following three equations:

$$
\begin{aligned}
& \frac{\partial^{2 \alpha} U_{1}(x, y, z)}{\partial x^{2 \alpha}} \\
& =\frac{\partial^{2} U_{1}(X, Y, Z)}{\partial X^{2}} \frac{\partial^{\alpha} X}{\partial x^{\alpha}}+\frac{\partial^{2} U_{1}(X, Y, Z)}{\partial Y^{2}} \frac{\partial^{\alpha} Y}{\partial x^{\alpha}}+\frac{\partial^{2} U_{1}(X, Y, Z)}{\partial Z^{2}} \frac{\partial^{\alpha} Z}{\partial z^{\alpha}} \\
& =\frac{\partial^{2} U_{1}(X, Y, Z)}{\partial X^{2}}, \\
& \frac{\partial^{2 \alpha} U_{2}(x, y, z)}{\partial x^{2 \alpha}} \\
& =\frac{\partial^{2} U_{2}(X, Y, Z)}{\partial X^{2}} \frac{\partial^{\alpha} X}{\partial x^{\alpha}}+\frac{\partial^{2} U_{2}(X, Y, Z)}{\partial Y^{2}} \frac{\partial^{\alpha} Y}{\partial x^{\alpha}}+\frac{\partial^{2} U_{2}(X, Y, Z)}{\partial Z^{2}} \frac{\partial^{\alpha} Z}{\partial z^{\alpha}} \\
& =\frac{\partial^{2} U_{2}(X, Y, Z)}{\partial X^{2}}, \\
& \frac{\partial^{2 \alpha} U_{3}(x, y, z)}{\partial x^{2 \alpha}} \\
& =\frac{\partial^{2} U_{3}(X, Y, Z)}{\partial X^{2}} \frac{\partial^{\alpha} X}{\partial x^{\alpha}}+\frac{\partial^{2} U_{3}(X, Y, Z)}{\partial Y^{2}} \frac{\partial^{\alpha} Y}{\partial x^{\alpha}}+\frac{\partial^{2} U_{3}(X, Y, Z)}{\partial Z^{2}} \frac{\partial^{\alpha} Z}{\partial z^{\alpha}} \\
& =\frac{\partial^{2} U_{3}(X, Y, Z)}{\partial X^{2}} .
\end{aligned}
$$

Finally, by taking into account Eqs. (8)-(10), we end up with (26).

\section{Wave equations on Cantor sets}

In this section, the applications of fractional complex transform method to handle threedimensional wave equations on Cantor sets are considered. Comparison between the fractional complex transform method, derived from modified R-L derivatives, and fractional complex transform via local fractional derivatives is investigated. 
Let us consider 3-D wave equation on Cantor sets (3). We now determine the fractional complex transform via local fractional derivatives

$$
\left\{\begin{array}{l}
T=\frac{t^{\alpha}}{\Gamma(1+\alpha)}, \\
X=\frac{(a x)^{\alpha}}{\Gamma(1+\alpha)}, \\
Y=\frac{(a y)^{\alpha}}{\Gamma(1+\alpha)}, \\
Z=\frac{(a z)^{\alpha}}{\Gamma(1+\alpha)}
\end{array}\right.
$$

such that

$$
\frac{\partial^{2} u(X, Y, Z, T)}{\partial T^{2}}+\nabla u(X, Y, Z, T)=0,
$$

where

$$
\nabla=\frac{\partial^{2}}{\partial X^{2}}+\frac{\partial^{2}}{\partial Y^{2}}+\frac{\partial^{2}}{\partial Z^{2}} .
$$

Let us consider the non-dimensional 2-D wave equation on Cantor sets.

We now determine the fractional complex transform via local fractional derivatives

$$
\left\{\begin{array}{l}
T=\frac{t^{\alpha}}{\Gamma(1+\alpha)}, \\
X=\frac{(a x)^{\alpha}}{\Gamma(1+\alpha)}, \\
Y=\frac{(a y)^{\alpha}}{\Gamma(1+\alpha)}
\end{array}\right.
$$

such that Eq. (6) is rewritten

$$
\frac{\partial^{2} u(X, Y, T)}{\partial T^{2}}+\nabla u(X, Y, T)=0
$$

where

$$
\nabla=\frac{\partial^{2}}{\partial X^{2}}+\frac{\partial^{2}}{\partial Y^{2}}
$$

If there is the mass function $[3-5,18,28]$

$$
\gamma^{\alpha}[F, a, b]=\frac{1}{\Gamma(1+\alpha)} H^{\alpha}(F \cap(a, b))=\frac{(b-a)^{\alpha}}{\Gamma(1+\alpha)},
$$

then we arrive at the following formula:

$$
\gamma^{\alpha}[F, l a, l b]=l^{\alpha} \frac{(b-a)^{\alpha}}{\Gamma(1+\alpha)} .
$$


Following the above results, we revisit the fractional complex transform method via local fractional derivatives, Eq. (12) are suggested by

$$
\left\{\begin{array}{l}
T(t)=\frac{t^{\alpha}}{\Gamma(1+\alpha)}=\gamma^{\alpha}[F, 0, t], \\
X(x)=\frac{(a x)^{\alpha}}{\Gamma(1+\alpha)}=\gamma^{\alpha}[F, 0, a x], \\
Y(y)=\frac{(a y)^{\alpha}}{\Gamma(1+\alpha)}=\gamma^{\alpha}[F, 0, a y], \\
Z(z)=\frac{(a z)^{\alpha}}{\Gamma(1+\alpha)}=\gamma^{\alpha}[F, 0, a z] .
\end{array}\right.
$$

From $[2-5,18,22,23]$, we conclude that

$$
\begin{array}{ll}
\left|T\left(t_{1}\right)-T\left(t_{2}\right)\right| \leq \varepsilon_{1}^{\alpha}, & \left|X\left(x_{1}\right)-X\left(x_{2}\right)\right| \leq \varepsilon_{2}^{\alpha}, \\
\left|Y\left(y_{1}\right)-Y\left(y_{2}\right)\right| \leq \varepsilon_{3}^{\alpha}, & \left|Z\left(z_{1}\right)-Z\left(z_{2}\right)\right| \leq \varepsilon_{4}^{\alpha}
\end{array}
$$

for any $0<\varepsilon_{i}$ and $\varepsilon_{i} \in R$, which implies that fractal dimensions of transferring pairs are $\alpha$.

\section{Conclusions}

In this manuscript, we consider that the fractional complex transform method is derived from the local fractional differential operator, which is set up on fractals. The obtained results on Cantor sets are related to physical phenomenon in Cantorian time-space. It is supposed that the transferring pairs are related to fractal measure that could be fundamental in understanding of the Cantorian monadic structure of quantum space time. The reported results have a potential application in observing the structure of differential equations from micro-physical to macro-physical behavior of substance in the real world.

\section{Competing interests}

The authors declare that they have no competing interests.

\section{Authors' contributions}

Authors contributed equally and in writing this article. Authors read and approved the final manuscript.

\section{Author details}

'School of Mechanical Engineering, Tianjin University, Tianjin, 300072, China. ${ }^{2}$ Institute of Medical Equipment, Academy of Military Medical Sciences, Tianjin, 300161, China. ${ }^{3}$ School of Mechanics \& Civil Engineering, China University of Mining \& Technology, Xuzhou, Jiangsu 221008, China. ${ }^{4}$ Institute of Software Science, Zhengzhou Normal University, Zhengzhou, 450044, China. ${ }^{5}$ Department of Mathematics, Faculty of Mathematical Sciences, University of Mazandaran, Babolsar, Iran. ${ }^{6}$ Department of Mathematics and Computer Sciences, Faculty of Arts and Sciences, Cankaya University, Ankara, 06530, Turkey. ${ }^{7}$ Department of Chemical and Materials Engineering, Faculty of Engineering, King Abdulaziz University, P.O. Box 80204, Jeddah, 21589, Saudi Arabia. ${ }^{8}$ Institute of Space Sciences, Magurele-Bucharest, Romania.

\section{Acknowledgements}

Dedicated to Professor Hari M Srivastava.

The authors would like to thank the editor and the referees for their useful comments and remarks. The work is supported by the Natural Science Foundation of Tianjin, China (No. 10JCZDJC25100).

Received: 23 January 2013 Accepted: 26 March 2013 Published: 10 April 2013

\section{References}

1. Kolwankar, KM, Gangal, AD: Hölder exponents of irregular signals and local fractional derivatives. Pramana J. Phys. 48(1), 49-68 (1997)

2. Kolwankar, KM, Gangal, AD: Local fractional Fokker-Planck equation. Phys. Rev. Lett. 80, $214-217$ (1998)

3. Yang, XJ: Advanced Local Fractional Calculus and Its Applications. World Science Publisher, New York (2012)

4. Yang, XJ: Local Fractional Functional Analysis and Its Applications. Asian Academic Publisher Limited, Hong Kong (2011)

5. Yang, XJ: Local fractional integral transforms. Prog. Nonlinear Sci. 4, 1-225 (2011)

6. Jumarie, G: The Minkowski's space-time is consistent with differential geometry of fractional order. Phys. Lett. A 363, 5-11 (2007) 
7. Jumarie, G: Modified Riemann-Liouville derivative and fractional Taylor series of non-differentiable functions further results. Comput. Math. Appl. 51, 1137-1376 (2006)

8. Parvate, A, Gangal, AD: Calculus on fractal subsets of real line - I: formulation. Fractals 17(1), 53-81 (2009)

9. Parvate, A, Gangal, AD: Fractal differential equations and fractal-time dynamical systems. Pramana J. Phys. 64, 389-409 (2005)

10. Chen, W: Time-space fabric underlying anomalous diffusion. Chaos Solitons Fractals 28, 923-929 (2006)

11. Chen, W, Zhang, XD, Korosak, D: Investigation on fractional and fractal derivative relaxation-oscillation models. Int. J. Nonlinear Sci. Numer. Simul. 11, 3-9 (2010)

12. Chen, W, Sun, HG, Zhang, XD, Koroak, D: Anomalous diffusion modeling by fractal and fractional derivatives. Comput. Math. Appl. 59, 1754-1758 (2010)

13. Adda, FB, Cresson, J: About non-differentiable functions. J. Math. Anal. Appl. 263, 721-737 (2001)

14. He, JH: A new fractal derivation. Therm. Sci. 15(1), 145-147 (2011)

15. He, JH: Asymptotic methods for solitary solutions and compactons. Abstr. Appl. Anal. 2012, 916793 (2012)

16. Fan, J, He, JH: Fractal derivative model for air permeability in hierarchic porous media. Abstr. Appl. Anal. 2012, 354701 (2012)

17. Hu, MS, Baleanu, D, Yang, XJ: One-phase problems for discontinuous heat transfer in fractal media. Math. Probl. Eng 2013, $358473(2013)$

18. Yang, XJ: Local fractional calculus and its applications. In: Proceedings of the 5th IFAC Workshop Fractional Differentiation and Its Applications, (FDA '12), pp. 1-8, Nanjing, China (2012)

19. Yang, XJ: Research on fractal mathematics and some applications in mechanics. M.S. thesis, China University of Mining and Technology (2009) (in Chinese)

20. Yang, XJ: A short note on local fractional calculus of function of one variable. J. Appl. Libr. Inf. Sci. 1(1), 1-13 (2012)

21. Zhong, WP, Gao, F, Shen, XM: Applications of Yang-Fourier transform to local fractional equations with local fractional derivative and local fractional integral. Adv. Math. Res. 461, 306-310 (2012)

22. Yang, XJ: Local fractional variational iteration method and its algorithms. Adv. Comput. Math. Appl. 1(3), 139-145 (2012)

23. Yang, XJ: Local fractional kernel transform in fractal space and its applications. Adv. Comput. Math. Appl. 1(2), 86-93 (2012)

24. Carpinteri, A, Sapora, A: Diffusion problems in fractal media defined on Cantor sets. Z. Angew. Math. Mech. 90, 203-210 (2010)

25. Carpinteri, A, Chiaia, B, Cornetti, P: Static-kinematic duality and the principle of virtual work in the mechanics of fractal media. Comput. Methods Appl. Mech. Eng. 191, 3-19 (2001)

26. Balankin, AS, Elizarraraz, BE: Map of fluid flow in fractal porous medium into fractal continuum flow. Phys. Rev. E 85(5), $056314(2012)$

27. Yang, XJ, Baleanu, D, Zhong, WP: Approximation solution to diffusion equation on Cantor time-space. Proc. Rom. Acad., Ser. A: Math. Phys. Tech. Sci. Inf. Sci. (2013, in press)

28. Hu, MS, Agarwal, RP, Yang, XJ: Local fractional Fourier series with application to wave equation in fractal vibrating string. Abstr. Appl. Anal. 2012, 567401 (2012)

29. Li, ZB, He, JH: Fractional complex transform for fractional differential equations. Math. Comput. Appl. 15(5), 970-973 (2010)

30. Li, ZB, He, JH: Application of the fractional complex transform to fractional differential equations. Nonlinear Sci. Lett. A, Math. Phys. Mech. 2(3), 121-126 (2011)

31. Li, ZB: An extended fractional complex transform. Int. J. Nonlinear Sci. Numer. Simul. 11, 0335-0337 (2010)

32. $\mathrm{Li}, \mathrm{ZB}, \mathrm{Zhu}, \mathrm{WH}, \mathrm{He}, \mathrm{JH}$ : Exact solutions of time-fractional heat conduction equation by the fractional complex transform. Therm. Sci. 16(2), 335-338 (2012)

33. He, JH: A short remark on fractional variational iteration method. Phys. Lett. A 375(38), $3362-3364$ (2011)

34. He, JH, Elagan, SK, Li, ZB: Geometrical explanation of the fractional complex transform and derivative chain rule for fractional calculus. Phys. Lett. A 376(4), 257-259 (2012)

35. Yang, XJ: The zero-mass renormalization group differential equations and limit cycles in non-smooth initial value problems. Prespacetime J. 3(9), 913-923 (2012)

36. Ibrahim, RW: Fractional complex transforms for fractional differential equations. Adv. Differ. Equ. 2012, 192-203 (2012)

37. Ibrahim, RW: Complex transforms for systems of fractional differential equations. Abstr. Appl. Anal. 2012, 814759 (2012)

38. Ghazanfari, B, Ghazanfari, AG: Solving system of fractional differential equations by fractional complex transform method. Asian J. Appl. Sci. (2012). doi:10.3923/ajaps.2012

39. Liangprom, A, Nonlaopon, K: On the convolution equation related to the diamond Klein-Gordon operator. Abstr. Appl. Anal. 2011, $908491(2011)$

doi:10.1186/1687-1847-2013-97

Cite this article as: Su et al.: Fractional complex transform method for wave equations on Cantor sets within local fractional differential operator. Advances in Difference Equations 2013 2013:97. 